\title{
Kernos
}

Revue internationale et pluridisciplinaire de religion

grecque antique

8 | 1995

Varia

\section{A. Moreau (éd.), L'Initiation. I: Les rites d'adolescence et les mystères, II: L'acquisition d'un savoir ou d'un pouvoir. Le lieu initiatique, parodies et perspectives}

\section{Edith Gilis}

\section{(2) OpenEdition}

Journals

Édition électronique

URL : http://journals.openedition.org/kernos/622

DOI : $10.4000 /$ kernos.622

ISSN : 2034-7871

Éditeur

Centre international d'étude de la religion grecque antique

Édition imprimée

Date de publication : 1 janvier 1995

Pagination : 313-318

ISSN : 0776-3824

\section{Référence électronique}

Edith Gilis, «A. Moreau (éd.), L'Initiation. I: Les rites d'adolescence et les mystères, II: L'acquisition d'un savoir ou d'un pouvoir. Le lieu initiatique, parodies et perspectives », Kernos [En ligne], 8| 1995, mis en ligne le 12 avril 2011, consulté le 22 septembre 2020. URL : http://journals.openedition.org/kernos/ 622 ; DOI : https://doi.org/10.4000/kernos.622 
aussi comme une puissance chthonienne, révélée par des pratiques de magicienne. Face à elle, Jason se présente comme un kouros passant par différentes épreuves d'initiation, qu'il réussit et qui lui valent comme récompense la royauté et un bieros gamos. L'histoire va modifier sensiblement ce schéma primitif, ainsi que le révèle la suite de l'analyse. Le voyage des Argonautes perd son aspect initiatique pour devenir une expédition colonisatrice, vantée quand les cités grecques s'assurent le contrôle commercial du bassin méditerranéen, critiquée amèrement, quand Athènes subit les contrecoups de sa politique impérialiste. Médée perd son aspect de divinité bienfaisante pour devenir une femme mauvaise et une sorcière redoutable; au terme de son évolution, elle retrouve sa puissance primitive, mais elle s'est transformée en puissance du mal. Face à elle, Jason perd son statut héroïque : devenant le protégé d'une femme et non plus d'une déesse, il est de ce fait dégradé et est voué à une déchéance inexorable. Dans une troisième partie enfin, Alain Moreau envisage différentes interprétations globales du mythe qui ont été proposées tantôt par les Anciens eux-mêmes (interprétation rationaliste), tantôt par nos contemporains (interprétations folkloriste, structuraliste, psychanalytique), pour montrer l'éclairage nouveau, mais aussi les limites de ces types de regard.

La lecture de ce livre non seulement affine notre vision du mythe du vanu-pied et de la sorcière, mais apporte de nombreux éléments de réflexions à propos d'œuvres particulières de l'Antiquité. On ne pourra plus aborder désormais l'étude de la Médée d'Euripide, de celle de Sénèque ou des différentes Argonautiques qui sont parvenues jusqu'à nous, sans tenir compte de la synthèse qu'Alain Moreau nous livre sur l'ensemble du mythe. Puisse le succès que rencontrera certainement cette étude le porter à soumettre d'autres légendes grecques à son investigation minutieuse et à son analyse critique et perspicace.

Monique MuND-DOPCHIE (Université Catholique de Louvain)

Alain Moreau (éd.), L'Initiation. I : Les rites d'adolescence et les mystères; II : L'acquisition d'un savoir ou d'un pouvoir. Le lieu initiatique, parodies et perspectives. Actes du colloque international de Montpellier, 11-14 avril 1991, Publications de la Recherche, Université Paul Valéry (Séminaire d'Étude des Mentalités Antiques), Montpellier III, 1992, 2 vol., $14,7 \times 21 \mathrm{~cm}, 326+318$ p. ISBN : 2-905397-45-4.

Les actes du colloque organisé par le Séminaire d'Étude des Mentalités Antiques sous l'égide d'Alain Moreau réunissent quarante communications. Organiser un colloque sur un tel sujet est qualifié par son organisateur « d'aventure dangereuse car rien n'est plus difficile à cerner que le mot "initiation" » (p, 6). C'est pourquoi les communications n'ont été acceptées que dans la mesure où elles pouvaient être intégrées dans l'une des trois catégories 
suivantes, dégagées à partir des études fondamentales de A. Van Gennep, $H$. Jeanmaire, $M$. Eliade et $J$. Ries, et qui participent toutes de la notion de «passage »: 1) initiation des adolescents (passage du monde de l'enfance à l'âge adulte); 2) initiation du myste (passage de l'état profane à celui d'initié, intégration à une secte); 3) initiation professionnelle (transformation de l'initié en chamane, acquisition d'un pouvoir exceptionnel).

Ces articles - dont on ne peut fournir ici qu'un résumé sommaire, vu leur nombre - ont été regroupés en dix chapitres.

\section{Rites archaiques}

Monique BILE (Les termes relatifs à l'initiation dans les inscriptions crétoises) étudie les rituels initiatiques proprement dits pour lesquels les sources littéraires complètent le témoignage des inscriptions, l'initiation étant l'étape indispensable à l'intégration du jeune garçon à la société des hommes adultes. - Après avoir comparé brièvement les Hyakinthies et les Panathénées, Pierre Brulé (Fêtes grecques: périodicité et initiations. Hyakintbies et Panatbénées) conclut que le noyau central de ces célébrations est constitué par un ensemble de rites de sortie de l'enfance des garçons et des filles. En outre, le côté conservateur de la société spartiate laisse penser que les Hyakinthies constitutent un modèle plus ancien que les Panathénées comme fête d'intégration de nouveaux initiés. - Bernard SERGENT (Celto-bellenica IV : la ruse) aborde un thème récurrent des épreuves initiatiques : la ruse dont l'initié doit faire preuve pour éviter un danger. C'est notamment le cas de deux récits présentant de traits initiatiques manifestes : la victoire de Cuchulainn contre Aiffé dans la littérature irlandaise et celle de Mélanthos contre Xanthos en Grèce. - Selon Dominique Briquel (Les légendes de fondation latines et l'initiation), le monde latin ne connaît plus la pratique de l'initiation systématique des jeunes gens; néanmoins de nombreuses légendes latines offrent un processus d'initiation, notamment les légendes des héros fondateurs.

\section{Homère}

Trois communications traitent de l'échec à l'initiation. La première est celle de Paul Wathelet (Rites de passage dans liliade : écbecs et réussites) qui s'interroge sur les allusions à des rites de passage qui apparaissent dans de nombreux épisodes de l'Iliade et notamment sur l'échec d'Achille dans son épreuve. Dans la deuxième, Ariadne GARTzIou-TATTI (Pâris-Alexandre dans lIliade) affirme que Pâris n'est pas un guerrier comme les autres. Éphèbe prolongé, qui meurt sur le champ de bataille, il n'a pas la possibilité de devenir un héros accompli caractérisé par la gloire impérissable. Dans la troisième, Evelyne SCHEID-Tissinier (Les prétendants de l'Odyssée, une génération perdue) considère cette génération intermédiaire entre celle, héroïque, d'Ulysse et celle de son fils, Télémaque, est vouée à la disparition. Quant à l'article d'A. Moreau (Odyssée, XXI, 101-139: l'examen de passage de Télémaque), il s'interroge sur la figure du fils d'Ulysse. Le jeune Télémaque ramène de son passage dans le Péloponnèse auprès de Nestor et de Ménélas une éducation; il 
est passé de l'enfance au monde adulte. Il suit un parcours initiatique analogue à celui de son père.

\section{Ambivalences et cas particuliers}

Juxtaposant Diodore et Pausanias, Colette Jourdarn-ANneQuin ( $\grave{A}$ propos d'un rituel pour Iolaos à Agyrion. Héraclès et l'initiation des jeunes gens) met en exergue l'ambivalence d'Héraclès : à la fois fondateur et initiateur d'un culte en l'honneur de Iolaos à Agyrion, accompagné de cérémonies initiatiques. - Gérard Capdeville ( La jeunesse de Camille) étudie une jeune héroïne recevant une initiation masculine qui en fait une vierge guerrière. Cette création littéraire de Virgile s'inspire des Amazones et d'autres motifs mythiques de la tradition grecque. - Dans le cadre des rites d'initiation réservé aux adolescents, Carlos Garcia Gual. (L'initiation de Daphnis et Chloé) suit les traces d'une initiation dans les mystères de l'amour. -- L'initiation n'étant pas exclusivement un thème de l'antiquité grecque, Lucette Roux (Les enfances de Sigismond dans la vie est un songe de Calderon) analyse cette oeuvre représentative du théâtre espagnol du XVIIe siècle. Apparaît dans le parcours initiatique des héros un mélange de mythologie antique et d'idéologie chrétienne.

\section{Des rites d'adolescence aux mystères: Dionysos}

Selon Jan Bremmer (Dionysos travesti), Dionysos, au moment où il apparaît en fille ou comme une fille, s'érige en dieu qui a montré sa puissance, en un dieu «adulte ». - Étudiant le thème de l'initiation dans le théâtre d'Euripide, György KarSAI (Tirésias dans les Bacchantes) analyse l'attitude de Tirésias. Celui-ci passe de l'état apollinien à l'état dionysiaque. Il veut devenir un initié du dieu et subit les étapes des rites initiatiques du culte de Dionysos, mais n'atteindra pas le but ultime de l'initiation. - Giovanni Casadio (Prébistoire de l'initiation dionysiaque) et Robert TuRCAN (L'élaboration des mystères dionysiaques à l'époque bellénistique et romaine : de l'orgiasme à l'initiation) s'attardent sur l'origine des mystères dionysiaques. Si pour le premier, l'origine du développement des mystères de Dionysos pourrait se trouver à Lerne, pour le second, l'initiation aux mystères que l'on peut vraiment qualifier de dionysiaques ne se développe qu'à l'époque hellénistique et à l'époque romaine.

\section{Les mystères}

Maria-Luisa SILVESTRE (L'initiation comme pratique politique dans les anciennes sociétés grecques selon les philosophes: Héraclite et les mystères d'Éphèse) s'interroge sur la réflexion philosophique et l'initiation. Cette réflexion admet-elle une interprétation qui réunisse le facteur religieux au facteur politique pour le rôle de l'initiation dans les sociétés grecques antiques ? Dans le cas d'Éphèse, Héraclite réagit violemment aux rites initiatiques sanglants car les adeptes des ces pratiques étaient du parti opposé à celui du philosophe. - L'Énéide, récit d'une initiation individuelle et d'une transformation spirituelle, est clairement analysée par Joël Thomas ( $L$ Énéide, récit initiatique). Les trois 
étapes du cheminement d'Énée correspondent aux trois degrés d'une initiation classique. - Gérard FREYBURGER (L'initiation pythagoricienne dans le Livre XV des Métamorphoses d'Ovide) explique l'attitude ambiguë du Pythagore des Métamorphoses d'Ovide par son comportement comme prêtre de la secte et s'appuie pour cela sur le parallélisme avec les prêtres d'Isis dans les Métamorphoses d'Apulée. L'isisme, doctrine pleine de grandeur, prête aussi aux sourires moqueurs. - Selon Nicole FICK (La métamorphose initiatique), dans l'œuvre d'Apulée, Lucius connaît une double métamorphose. Victime de sa sensualité, il tombe dans l'animalité, pour en ressortir peu à peu et redevenir un homme grâce à Isis. Initiation isiaque, mais plus encore philosophique et esthétique, dans l'esprit de Platon. - La cérémonie de l'initiation chrétienne étudiée par Paul FORCE (Place et signification de la Redditio Symboli dans l'initiation chrétienne des premiers siècles de l'Église) est l'initiation baptismale considérée comme culte à mystère à valeur eschatologique, car elle comportait un aspect secret. - Francis Dubost (Aspects initiatiques de la littérature de Graal) attache sa réflexion au thème de l'initiation mystique par excellence, la quête du Graal. Selon lui, quelles que soient les versions littéraires de cette quête, la recherche du Graal constitue une initiation, le passage vers la sainteté. Néanmoins le Graal n'est pas le véritable objet de la quête, mais le réceptacle de divers contenus initiatiques.

\section{Les professionnels}

Didier Pralon (Les travaux d'Héraclès dans lHéraclès furieux d'Euripide) envisage le héros initiateur et initié par excellence, Héraclès. Le classement des travaux met en valeur ses qualités d'initié à la chasse, à la guerre et à l'immortalité. - À partir de documents figurés et d'inscriptions, Noris LyAPOUSTINE (Rites de passage dans le monde artisanal à Pompéi lier siècle ap. J.-C.D, se penche sur l'initiation aux métiers de l'artisanat à Pompéi. - Fritz GRAF (Comment devenir un magicien? Le rituel d'initiation magique grécoégyptien) cerne, au départ de divers papyri, les rituels initiatiques du magicien, ainsi que l'interprétation que les sorciers donnent de leurs pratiques à la lumière de l'expérience des mystères. - Selon Michèle Brraud (Parcours initiatique dans les Merveilles d'au delà de Thulé), différentes initiations se déroulent dans cette œuvre: l'adolescente Dercyllis suit une initiation de jeunesse (exil, pérégrination...) aidé en cela par un initiateur, Astraios, mais qui est lui-même en quête d'un savoir supérieur. Deinias quant à lui découvre les anomalies du temps cosmique, l'abolition magique de l'espace n'est pas sans rappeler le vol magique des «chamans» grecs (Dodds). - Spécialiste des peuplades indiennes, Danièle VAzeILles (Initiations chamaniques en Amérique du Nord: le cas des Indiens sioux Lakotas) pense que dans la société des Indiens sioux, il est nécessaire d'entrer en contact avec les esprits, en pratiquant soit des rituels collectifs, soit en devenant l'«élu ». Pour les Sioux actuels, ces rites traditionnels sont devenus un moyen d'affirmer leur identité culturelle. 


\section{L'écrivain comme initié et initiateur}

Deux articles sont consacrés à un écrivain considéré comme un spécialiste en initiation : Hésiode. Le premier, de Marie-José Benejam-Bontems, (Les Travaux et les Jours d'Hésiode : un poème initiatique), affirme que le mythe des races est l'enseignement initiatique donné par un poète à l'humanité : "l'homme de fer» peut se régénérer et devenir «l'homme d'or » qui « vit comme les dieux ». Le poète devient lui-même initiateur. Le second, de Bernard DEFORGE (Hésiode: initiation et nom), prétend que dans le prologue de la Théogonie est rapportée l'initiation d'Hésiode par les Muses, expérience personnelle authentique s'exprimant notamment dans le statut des mots et des noms. L'auteur présente un parallèle avec un poème babylonien. - MarieLouise AUDIN (Métamorpbose d'un mythe de cbâtiment en mythe initiatique : le Rocher de Sisyphe de Roger Callois) analyse l'actualisation des mythes grecs anciens. Chez Roger Callois, le mythe du châtiment de Sisyphe devient un mythe initiatique éclairé par les inquiétudes contemporaines.

\section{Le lieu initiatique}

En Grèce ancienne, selon Claude CALAME (Prairies intouchées et jardins d'Aphrodite : espaces « initiatiques » en Grèce), les rites relevant de l'initiation tribale ne se tiennent pas nécessairement dans un espace sauvage, non cultivé, mais dans un paysage composé comme le jardin. L'auteur suggère également des formes différentes de jardin qui déterminent diverses fonctions initiatiques. - La mer, lieu initiatique privilégié, entre sauvagerie et culture, est étudiée par Hervé DuCHeNe (Initiation et élément marin en Grèce ancienne). Selon lui, l'eau a une valeur purificatrice mais joue aussi un rôle important dans les grands récits initiatiques (Ulysse, Thésée, ...). - Christine GoYENs-Slezakowa montre bien dans son article (L'initiation dans Philoctète de Sopbocle) que les îles, dans le cas présent, Lemnos, sont des endroits sauvages propres à accueillir un futur initié. Il est admis de tous que le fils de Poéas y connait une initiation, mais de quel type ? L'auteur conclut - avec raison - que l'initiation de Philoctète est celle d'un futur « chaman», - L'originalité de la communication de Richard Buxton (Le centaure : l'espace initiatique dans Henry IV de Shakespeare) réside dans le fait qu'un fils de roi est initié à la fonction dont il héritera dans le quartier ensauvagé de la ville de Londres, et plus précisément dans la taverne-caverne d'Eascheap. Le futur Henry $V$ abandonnera la sauvagerie de sa jeunesse pour s'intégrer dans le monde civilisé.

\section{Parodies}

Les rites d'initiation sont souvent des rites de passage liés à la jeunesse. L'originalité d'Aristophane selon Michel Menu (Pbiloclélon: une initiation de la vieillesse dans les comédies d'Aristophane ?) tient à ce qu'il propose en apparence une initiation de la vieillesse. Mais cette initiation est burlesque, associée à tous les excès de la société contemporaine. L'initiation chez le célèbre comique grec est encore traitée par Marie-Laure FreYburger, (Aristophane: 
l'initiation dévoilée et parodiée). Dans les Nuées, Aristophane s'en prendrait, selon l'auteur, autant à l'orphisme de Socrate qu'à ses conceptions sophistiques. Ceci pour dire que ces cultes sont dangereux car ils remettent en cause les cultes traditionnels de la religion officielle de la fin du Ve siècle. - Gérard GoUIRAN (L'initiation dans le Roman de Jaufré) aborde la littérature initiatique du moyen âge. Roman arthurien, le roman de Jaufré parodie les thèmes chevaleresques, mais constitue néanmoins l'initiation du héros, doublé d'une réorganisation du monde.

\section{L'initiation aujourd'bui}

Le thème de l'initiation n'a pas disparu de nos sociétés modernes, elle est même encore fort prisée. Ainsi notamment dans les films westerns, comme le développe Henri AgrL (Le rite d'initiation dans le western). Le thème initiatique prépondérant dans ce cas-ci est celui du jeune garçon qui doit s'affirmer en adulte, aidé en cela par un homme d'âge mûr, à la fois gourou et protecteur, qui se retire une fois la mission accomplie. - De nos jours, le danger de la disparition de tout rituel «initiatique » entraîne de graves désordres chez les adolescents et les jeunes adultes. Marie-José BATAILle (Initiation et psychanalyse) attire notre attention sur le fait que sans point de repère, ces jeunes sont attirés par des sectes ou des reviviscences mystiques néo-nazies. Selon l'auteur, la psychanalyse peut remédier à cette absence et être assimilée à un processus initiatique. - Dans la dernière communication, Simone VIERNe (Le mythe initiatique aujourd'bui), conclut que même dans nos sociétés désacralisées le mythe initiatique est un élan créateur. Sa charge imaginaire est indispensable à l'homme pour répondre à l'angoisse de sa condition mortelle.

Ce volume d'actes est remarquable, tant au plan matériel qu'au plan scientifique. La variété des thèmes exploités (antiquité grecque, monde romain et celtique, littérature médiévale et espagnole...) et la diversité des disciplines utilisées (inscriptions, textes historiques et littéraires ...) au cours de ce colloque donnent une information complète sur les derniers développements de la recherche en matière d'initiation.

Edith GILIS

(Université de Liège)

John H. OAkLey, Rebecca H. Sinos, The Wedding in Ancient Atbens, Madison, The University of Wisconsin Press, 1993. 1 vol. 22 x $28,5 \mathrm{~cm}$, XIV+153 p., 130 fig. (Wisconsin Studies in Classics). ISBN : 0-299-13720-1.

Parmi les rituels de passage, le mariage est sans doute l'un des plus importants, pour les individus et pour la communauté. Caractérisé par des rites de séparation, de transition et d'incorporation bien connus depuis l'étude de Van Gennep sur les rites d'initiation, le temps des noces offre à l'analyse un symbolisme très riche qui permet d'appréhender à la fois le statut du couple et la nature de l'union. Les noces se situent donc au cœur de ce livre qui, pour éviter de recréer in abstracto à partir de sources très disparates une cérémonie 